\title{
Prostate cancer age-standardised incidence increase between 2006 and 2016 in Gauteng Province, South Africa: A laboratory data-based analysis
}

\author{
N Cassim, ${ }^{1,2}$ MPH; A Ahmad, ${ }^{3}$ MB ChB, MMed (Urology); R Wadee, ${ }^{2,4}$ MB BCh, FC Path (SA) Anat, MMed (Anatomical Pathology), PhD; \\ T R Rebbeck, ${ }^{5}$ PhD; D K Glencross, ${ }^{1,2}$ MB ChB, MMed (Haematology); J A George, ${ }^{6}$ MB BCh, MSc, FC Path (Clinical Pathology), PhD \\ ${ }^{1}$ Department of Molecular Medicine and Haematology, Faculty of Health Sciences, University of the Witwatersrand, Johannesburg, South Africa \\ ${ }^{2}$ National Health Laboratory Service, Johannesburg, South Africa \\ ${ }^{3}$ Department of Urology, Faculty of Health Sciences, University of the Witwatersrand, Johannesburg, South Africa \\ ${ }^{4}$ Department of Anatomical Pathology, Faculty of Health Sciences, University of the Witwatersrand, Johannesburg, South Africa \\ ${ }^{5}$ Harvard T H Chan School of Public Health and Dana Farber Cancer Institute, Boston, USA \\ ${ }^{6}$ Department of Chemical Pathology, Faculty of Health Sciences, University of the Witwatersrand, and National Health Laboratory Service, \\ Johannesburg, South Africa
}

Corresponding author: $N$ Cassim (naseem.cassim@wits.ac.za)

\begin{abstract}
Background. Prostate cancer (PCa) is the leading male neoplasm in South Africa (SA) and is the second most frequently diagnosed cancer among men globally. Age-specific incidence rates (ASIRs) vary by up to 189-fold globally, with an ASIR of 68.0 per 100000 in 2018 in SA. Objectives. To describe PCa among men undergoing prostate biopsy in Gauteng Province, SA.

Methods. We undertook a retrospective descriptive study using prostate biopsy data collected from the National Health Laboratory Service (NHLS) database between 2006 and 2016. We extracted the Systematized Nomenclature of Medicine (SNOMED) clinical terms morphology and topography codes to assign histological findings using the International Classification of Diseases for Oncology. PCa was defined as adenocarcinoma with a reported Gleason Score (GS). The new grade group (GG) based on the GS is defined as follows; (i) GG1 for a GS <6; (ii) GG2 for a GS of $3+4=7$; (iii) GG3 for a GS of $4+3=7$; (iv) GG4 for a GS of 8; and ( $v$ ) GG5 for a GS $\geq 9$. Higher-grade disease was defined as GG4 and GG5 (GS $\geq 8$ ), in line with local guidelines. We reported associations of PCa with a GS $\geq 7$ with age and race and used provincial and world standard population data to determine annual ASIRs.

Results. We identified 22937 biopsies referred to the NHLS between 2006 and 2016. Of the 6448 biopsies (39\%) with a PCa finding for black Africans, $46 \%$ were diagnosed with high-risk PCa compared with $36-40 \%$ for other race groups $(p<0.0001)$. Black Africans were more likely than whites to have GG4 or GG5 PCa (odds ratio 1.45; 95\% confidence interval 1.27 - 1.67). The ASIR increased from 44.9 per 100000 in 2006 to 57.3 per 100000 in 2016.

Conclusions. Black African men were significantly more likely to present with PCa with a GS $\geq 8$ (GG4 and GG5) compared with the other racial groups in Gauteng. The ASIR increased dramatically during the study period, perhaps as a result of increased screening and awareness. There is a need for additional research to better understand why black African men present with higher-grade disease.

S Afr Med J 2021;111(1):26-32. https://doi.org/10.7196/SAMJ.2021.v111i1.14850
\end{abstract}

Prostate cancer (PCa) is becoming a major public health concern as life expectancy increases globally. ${ }^{[1,2]}$ In 2018, the global PCa incidence was the second highest after lung cancer. ${ }^{[3]}$ PCa incidence varied by up to 189 -fold across 186 countries, with age-standardised incidence rate (ASIR) ranges varying widely from 1.0 per 100000 in Bhutan to as high as 189.1 in Guadeloupe in 2018. ${ }^{[4]}$ Comparatively higher ASIRs were reported for some less-developed regions such as Barbados (129.3 per 100 000), Bahamas (85.8), South Africa (SA) (68.0) and Reunion (63.7). ${ }^{[4]}$ The PCa ASIR is highest among men of African descent, reflecting racial and genetic predisposition in these countries. ${ }^{[5]}$ The varying PCa ASIR could also be due to varying PCa screening and testing patterns that affect incidence. ${ }^{[6]}$

Limited SA data reported that black Africans present with more aggressive PCa, with a Gleason Score (GS) $\geq 7$ reported in $36 \%$, compared with $17 \%$ for African Americans. ${ }^{[7]}$ The GS is the strongest prognostic factor for PCa treatment. ${ }^{[8]}$ Local guidelines categorise PCa risk using the GS as follows: (i) 2 - 6 low; (ii) 7 intermediate; and (iii) $8-10$ high. ${ }^{[9,10]}$ The new grade group (GG) is based on the
GS as follows: (i) GG1 for a GS $\leq 6$; (ii) GG2 for a GS of $3+4=7$; (iii) GG3 for a GS of $4+3=7$; (iv) GG4 for a GS of 8; and (v) GG5 for a GS $\geq 9 .{ }^{[9,11]}$ Limited PCa biopsy data exist for black African men, however, with most local studies reporting data for selected health facilities. ${ }^{[7,12,13]}$ Currently, aside from local cancer registry data, which reported national 2014 data by 2018, there are no comprehensive public sector PCa data for Gauteng Province. ${ }^{[14]}$

In SA, PCa is the leading neoplasm in men. ${ }^{[3,4]}$ Risk factors include age, race (black African) and family history. ${ }^{[1,9,10,15]}$ The National Health Laboratory Service (NHLS) provides diagnostic services to $80 \%$ of the population. ${ }^{[16]}$ Routinely accumulated laboratory data through analysis of diagnostic samples offer a unique opportunity to describe PCa histological characteristics with the related demographic detail in local populations. The Systematized Nomenclature of Medicine (SNOMED) clinical terms (CT) topography (T) and morphology $(\mathrm{M})$ codes captured routinely based on the histological findings could be used to represent relevant clinical information consistently and comprehensively as an essential part of producing 
electronic health information. ${ }^{[17]}$ The $\mathrm{T}$ code identifies anatomical terms for each organ, and the $\mathrm{M}$ code describes the microscopic changes observed. ${ }^{[17]}$

We chose Gauteng for our study because it is home to $24.1 \%$ of the national population ${ }^{[18]}$ and represents a typical dense urban area. It is the economic hub of the country with the highest numbers of both international and domestic migrants, the latter originating from provinces such as Limpopo, KwaZulu-Natal and Eastern Cape. ${ }^{[18,19]}$ These domestic migrants are aged between 15 and 64 years, with males outnumbering females by 111 to $100 .{ }^{[20]}$ Over $70 \%$ of migrants are black Africans. ${ }^{[20]}$

\section{Objectives}

To describe PCa among men undergoing prostate biopsies in Gauteng Province, SA, using data fields directly extracted from patient laboratory records.

\section{Methods \\ Study design}

We extracted all prostate biopsies done on men aged $>30$ years between 1 January 2006 and 31 December 2016 and referred to the NHLS for pathology evaluation in Gauteng. Two data sets were extracted from the national laboratory data repository that houses laboratory information system (LIS) collated patient laboratory reports. ${ }^{[16]}$ These data sets were: (i) narrative prostate biopsy data; and (ii) SNOMED CT code(s). Ethical clearance was obtained from the University of the Witwatersrand, Johannesburg (ref. no. M170419).

\section{Biopsy data preparation}

The narrative biopsy extract included the following variables: $(i)$ episode number; (ii) unique patient identifier; (iii) age; (iv) gender; $(v)$ racial group; (vi) facility name; (vii) reviewed date; and (viii) biopsy narrative report. The episode number identifies samples for all laboratory processes. The unique patient identifier was generated using the organisation probabilistic matching algorithm that includes fuzzy logic matching, minimising the duplication of PCa case reports. ${ }^{[21]}$ For duplicate records, only the first record was used to calculate the ASIR. A sequence number was generated for chronologically sorted patient data. The GS was manually coded, with a random sample independently verified to validate manual coding $(n=369)$.

The SNOMED CT data extract included the episode number and the $\mathrm{M}$ and $\mathrm{T}$ codes. The $\mathrm{M}$ code describes the microscopic changes noted in cells, tissues, and organs. ${ }^{[17]}$ The $\mathrm{T}$ code describes the anatomical site of origin (prostate). ${ }^{[17]}$ We used the SNOMED $\mathrm{CT}$ codes to develop lookup tables to report: (i) histological findings (PCa/not PCa); (ii) biopsy findings; (iii) tumour type; and (iv) organ, with the assistance of an experienced anatomical pathologist and urologist (Appendix A, http://samj.org.za/public/sup/14850.png). We used the International Classification of Diseases for Oncology to assign PCa findings, i.e. adenocarcinoma tumour type with the associated tumour grading (GS). ${ }^{[22]}$ For missing $M$ codes, we manually coded biopsies using the narrative result text.

Racial group was identified as an important PCa risk factor. ${ }^{[7,12,13]} \mathrm{As}$ racial group was poorly recorded ( $94.5 \%$ missing), we used a locally developed hot-deck imputation method to assign missing data, ${ }^{[23]}$ described in an unpublished study (Chen W, Kellett P, Greyling M, Sengayi M, 'The use of surnames to impute missing ethnicity data in the National Cancer Registry database of South Africa, 2019). Well-populated cancer registry data with patient-reported race grouping were used to construct the imputation reference panel. The racial groups reported by Statistics South Africa in the 2011 census $^{[24]}$ are Indian/Asian, black African, coloured (mixed ancestry from European, Indian subcontinent and local indigenous African peoples $^{[25]}$ ) and white.

We used a relational database to combine the respective tables using a left outer join with the narrative biopsy data extract as the master table (Appendix A, database diagram showing how data from the SNOMED CT lookup tables and the race imputation data sets were used to create the prostate cancer data for this study, http://samj. org.za/public/sup/14850.png). Data were prepared using Microsoft Office Professional Plus 2013 (Microsoft, USA) and analysed using SAS 9.4 (SAS Institute, USA) and Stata 12 (StataCorp, USA).

Descriptive statistics by racial group were: (i) mean age (with range); (ii) biopsy numbers; (iii) biopsy findings; and (iv) number of biopsies reporting a PCa histological finding. We compared the mean age across racial groups by one-way analysis of variance (ANOVA). We used the $\chi^{2}$ test to identify whether there was a statistical difference for age category and biopsy findings for the four race groups. An alpha level of 0.05 was used for all association tests.

We used a pyramid chart to report the number of biopsies with a PCa and non-PCa finding for age categories (excluding unknown age). Age was reported on the $x$-axis and percentage of biopsies on the $y$-axis. Annual biopsy numbers were reported as a bar chart.

A multiple logistic regression method was used to determine the factors associated with PCa with a GG $\geq 4$, controlling for age and racial group. This is in line with local guidelines for a GS $\geq 8$ classified as high risk. ${ }^{[9,10]}$ We reported odds ratios (ORs), $p$-values and confidence intervals (CIs).

Biopsies with a PCa finding were analysed by GS and GG risk group for the four racial groups, reported as a stacked bar chart. ${ }^{[9,10]}$ We used a stacked bar chart to report the percentage of biopsies per year for the GS and GG risk categories, with GG4 and GG5 reported together.

The ASIR per 100000 and average annual percentage change (AAPC) were calculated for defined age categories for each calendar year using the Joinpoint trend analysis software. Data variables for the model were: (i) gender; (ii) year of diagnosis; (iii) age categories/ groups; (iv) number of incident cases (count variable); $(v)$ population estimates; and (vi) Segi world standard population. ${ }^{[26-28]}$ The last was used to enable comparison with cancer registry reports.

\section{Results}

There were 25010 biopsies extracted from the LIS. The SNOMED CT codes were used to assign $90 \%$ of histological findings, with $10 \%$ manually coded. We excluded 285 biopsies that did not meet the inclusion criteria, and excluded a further 2073 non-prostatic biopsies (8.3\%). The total sample size was therefore 22937 . It was not possible to determine a GS for $455 / 8390$ biopsies (5.4\%) with a PCa finding. We randomly reviewed GSs for accuracy and noted no misclassification errors.

\section{Prostate biopsy outcomes by age and racial group}

The mean age by racial group was 66.3 (range 31 - 92) years for Indians/Asians, 67.4 (30 - 99) years for black Africans, and 67.7 (30 99) years for whites (Table 1). A statistically significant difference for mean age for the four racial groups was determined by one-way ANOVA $(p<0.001)$. There was a significant difference in biopsy frequency by age category across the four racial groups $(p<0.0001)$. A malignant biopsy finding was reported for $46 \%$ of black Africans compared with $39 \%, 37 \%$ and $30 \%$ of coloureds, whites and Indians/ Asians, respectively. For black Africans, 39\% of biopsies reported a PCa finding, compared with 23\%, 29\% and 31\% for Indians/Asians, whites and coloureds, respectively $(p<0.0001)$.

Twenty-three percent of biopsies from the 65 - 69-year age category reported a PCa finding, as opposed to $19 \%$ and $20 \%$ for the $60-64$ - 


\begin{tabular}{|c|c|c|c|c|}
\hline & Indian/Asian & Black African & Coloured & White \\
\hline Biopsy samples, $n$ & 487 & 16491 & 1129 & 3422 \\
\hline Age (years), mean (range) & $66.3(31-92)$ & $67.4(30-99)$ & $67.4(35-99)$ & $67.7(30-99)$ \\
\hline$p$-value ${ }^{*}$ & & & & $<0.001$ \\
\hline \multicolumn{5}{|l|}{ Age category (years), $n(\%)$} \\
\hline $30-39$ & $4(1)$ & $49(0)$ & $2(0)$ & $7(0)$ \\
\hline $40-49$ & $13(3)$ & $298(2)$ & $22(2)$ & $44(1)$ \\
\hline $50-59$ & $76(16)$ & $2418(15)$ & $160(14)$ & $436(13)$ \\
\hline$\geq 60$ & $380(78)$ & $12502(76)$ & $890(79)$ & $2830(83)$ \\
\hline Unknown & $14(3)$ & $1224(7)$ & $55(5)$ & $105(3)$ \\
\hline$p$-value ${ }^{\dagger}$ & & & & $<0.0001$ \\
\hline \multicolumn{5}{|l|}{ Biopsy findings, $n(\%)$} \\
\hline Atypia/dysplasia & $21(4)$ & $575(3)$ & $43(4)$ & $129(4)$ \\
\hline Benign/negative for malignancy & $317(65)$ & $8321(50)$ & $641(57)$ & $2031(59)$ \\
\hline Neoplasm, malignant & $147(30)$ & $7561(46)$ & $442(39)$ & $1255(37)$ \\
\hline Uncertain whether benign or malignant & $2(0.5)$ & $34(0)$ & $3(0)$ & $7(0)$ \\
\hline$p$-value ${ }^{\dagger}$ & & & & $<0.0001$ \\
\hline Prostate cancer, $n(\%)^{\ddagger}$ & $111(23)$ & 6448 (39) & $354(31)$ & $1009(29)$ \\
\hline
\end{tabular}

and 70 - 74-year age categories, respectively (Fig. 1). The percentage of non-PCa biopsies was lowest in older ages, with $12 \%$ reported in the $75-79$-year and $8 \%$ in the $\geq 80$ year age categories. Eleven percent and $5 \%$ of biopsies reported a PCa finding for the 50-54-year and 55-59-year age categories, respectively. A non-PCa finding was reported for $22 \%$ of biopsies in the 60 64 -year age category, decreasing to $19 \%$ and $11 \%$ for the 65 - 69 -year and $70-74$-year age categories, respectively.

Black Africans were more likely to have PCa with a GG $\geq 4$ than whites (OR 1.45; 95\% CI 1.27 - 1.67) (Table 2). Similarly, coloureds and Indians/Asians were also more likely to have PCa with a GG $\geq 4$ than whites, with ORs of 1.14 (95\% CI 0.89 $1.46)$ and 1.06 (95\% CI 0.71 - 1.59), respectively.

Men aged $<70$ years were determined to have a reduced probability of being diagnosed with PCa with a GG $\geq 4$ compared with those aged $\geq 70$ years, with an $\mathrm{OR}$ of 0.99 (95\% CI $0.69-1.42)$ for ages 30 49 years, 0.90 (95\% CI $0.79-1.03)$ for 50 59 years, and 0.87 (CI 0.79 - 0.96) for 60 69 years. Controlling for racial group, males aged $\geq 70$ years were at a significantly greater risk of having PCa with a GG $\geq 4$.

Between 2006 and 2016, the number of biopsies increased from 1630 to 2761 (Fig. 2). We noted lower volumes in 2008 ( $n=1$ 168), decreasing from 1610 in 2007 ( $27 \%$ decrease). A $25 \%$ increase in biopsies was reported between $2010 \quad(n=1868)$ and $2011 \quad(n=2335)$, compared with 19\% between $2014(n=2357)$ and $2015(n=2805)$.

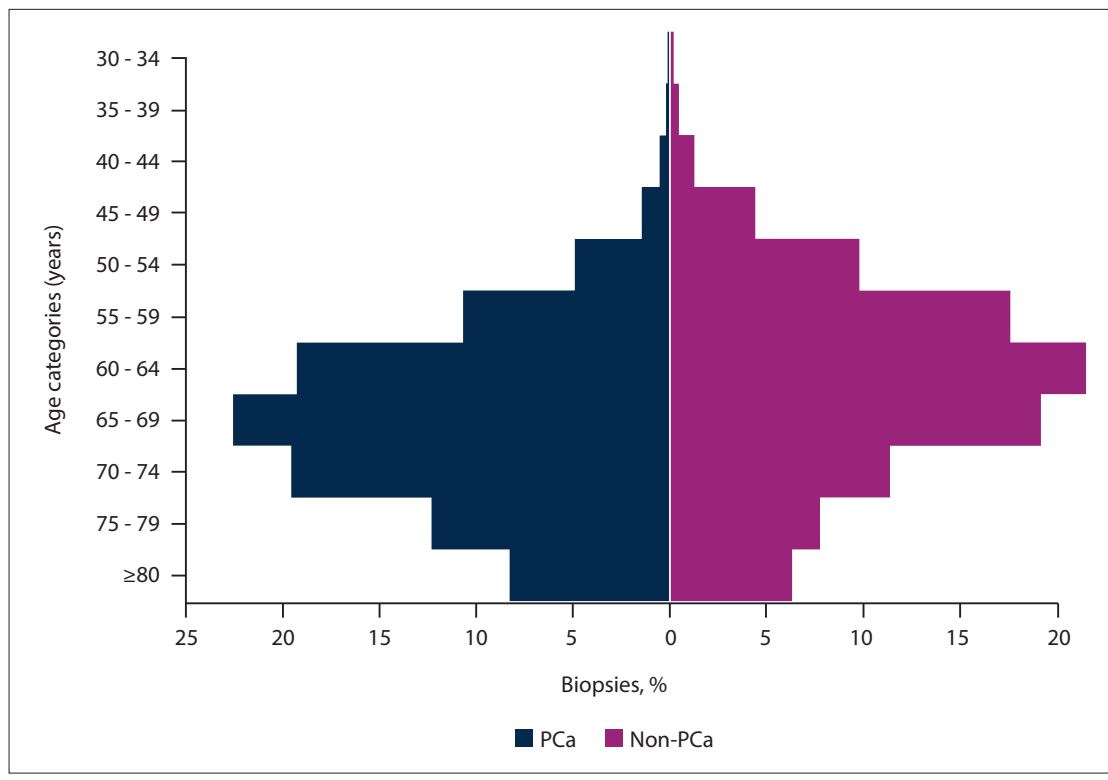

Fig. 1. Age distribution for PCa findings. Biopsy numbers reported by age category for both PCa and non-PCa histological findings in Gauteng Province between 2006 and 2016, as a population pyramid. $(P C a=$ prostate cancer. $)$

\section{Analysis of low-, intermediate- and high-risk GSs}

A high-risk GS $(\geq 8)$ was reported for $46 \%$ of biopsies ( $n=6448)$ with a PCa finding for black Africans compared with 40\%, 38\% and $36 \%$ for coloureds, Indians/Asians and whites, respectively (Fig. 3). The percentage of biopsies with a GG2 ranged from 17\% for whites to $24 \%$ for coloureds. Similarly, the percentage of biopsies with a GG3 ranged from $10 \%$ for coloureds to $15 \%$ for Indians/ Asians. Of biopsies for black Africans, GG2 and GG3 were reported in $18 \%$ and $13 \%$, respectively.
Between 2006 and 2016, the percentage of biopsies with a GG1 decreased from $27 \%$ to $18 \%$ (Fig. 4). Conversely, the percentage of biopsies with a GG $\geq 4$ increased from $42 \%$ in 2006 to $48 \%$ by 2016 . Biopsies with a GG3 increased from $12 \%$ in 2006 to $27 \%$ by 2016 . Conversely, the percentage of biopsies with a GG2 decreased from $20 \%$ to $18 \%$ over the same time period.

\section{ASIRs for prostate cancer}

The ASIR increased from 44.92 per 100000 in 2006 to 57.31 by 2016 (Fig. 5). Between 2006 and 2016, we reported an AAPC of 2.7\% 
(95\% CI - $1.2-6.7)$, which was significant $(p<0.05)$. A lower ASIR was reported for 2008 (27.0 per 100 000). Between 2014 and 2015, the ASIR increased from 40.8 to 52.6 per 100 000, respectively (largest year-onyear increase).

Between 2006 and 2016, an annual percentage change (APC) and average annual percentage change (AAPC) of 2.7 (95\% CI -1.2 - 6.7) was reported (Table 3). The $p$-value was $<0.0001$ for both the APC and AAPC, indicating a statistical difference.

\section{Discussion}

Our study reported an increase in biopsy numbers between 2006 and 2016, with PCa diagnosed predominantly in older men. The analysis also reveals that the ASIR in Gauteng Province has increased substantially from 44.9 (2006) to 57.3 (2016) per 100000. We also found that black Africans were statistically significantly more likely to have advanced disease with a GG $\geq 4$ compared with other racial groups.

The large increase in prostate biopsies reported in our study may indirectly reflect

Table 2. Multiple logistic regression analysis to assess the association between prostate cancer outcomes, GS, racial group and age category ${ }^{*}$

\begin{tabular}{|c|c|c|c|}
\hline Characteristic & OR & 95\% CI & $p$-value \\
\hline \multicolumn{4}{|l|}{ Racial group } \\
\hline White & 1 & - & - \\
\hline Indian/Asian & 1.06 & $0.71-1.59$ & 0.764 \\
\hline Black African & 1.45 & $1.27-1.67$ & $<0.0001$ \\
\hline Coloured & 1.14 & $0.89-1.46$ & 0.311 \\
\hline \multicolumn{4}{|c|}{ Age category (years) } \\
\hline $30-49$ & 0.99 & $0.69-1.42$ & 0.948 \\
\hline $50-59$ & 0.90 & $0.79-1.03$ & 0.131 \\
\hline $60-69$ & 0.87 & $0.79-0.96$ & 0.005 \\
\hline$\geq 70$ & 1 & - & - \\
\hline
\end{tabular}

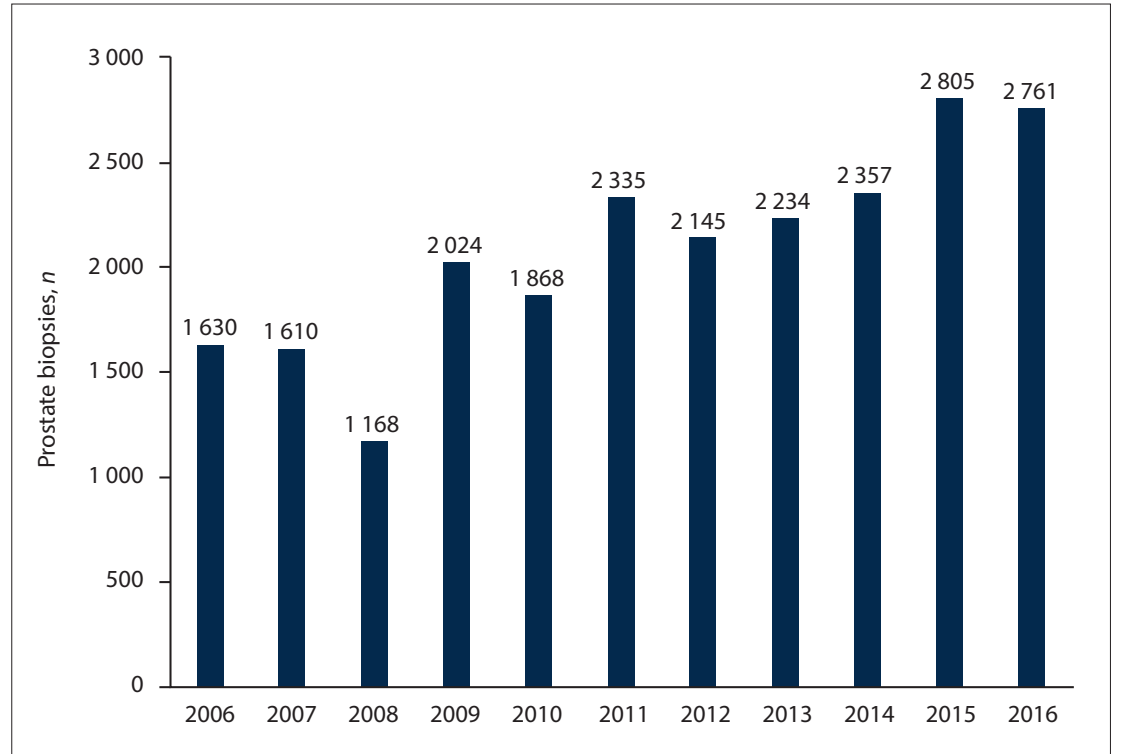

Fig. 2. Prostate biopsy numbers for Gauteng Province between 2006 and 2016.

improved access to care. This would include more patient referrals to a urologist following an elevated prostate-specific antigen (PSA) level and/or abnormal findings on digital rectal examination. Urology services that were previously limited to academic centres have been introduced in several provincial hospitals, ${ }^{[26]}$ so patients can be managed locally without the need to travel to tertiary hospitals.

Our findings of an increasing ASIR are in keeping with those of Babb et al., ${ }^{[27]}$ who reported a rise in SA from 17 per 100000 in 1986 to 27 per 100000 by 2006. Similarly, between 2007 and 2013, the National Cancer Registry (NCR) reported that the ASIR increased from 29 to 44 per $100000 .{ }^{[28,29]}$ We have shown a slightly higher increase compared with Babb et al. ${ }^{[27]}$ and the NCRreported national values. ${ }^{[30]}$ Between 2007 and 2013, the PCa ASIR in Gauteng was substantially higher than national estimates at 41.1 v. 29 per 100000 and 48.4 v. 44 per 100000 , respectively. ${ }^{[28,29]}$ By reporting provincial trends, we are able to highlight areas with an ASIR increasing faster than nationally reported data. Some of the reasons for the reported increase in incidence include improved urological guidelines, better access to screening, enhanced patient awareness, and improved medical aid coverage for PCa diagnosis. ${ }^{[9,10]}$

The 2013 PCa urology treatment guidelines recommend screening from the age of 40 for black Africans and 45 for all males. ${ }^{[10]}$ Studies have shown that PSA screening is associated with increased diagnosis and decreased mortality. ${ }^{[8]}$ Our own unpublished data (Cassim N, Ahmad A, Glencross DK, George JA, 'Trends in prostate specific antigen (PSA) testing for primary health care facilities in the Gauteng province, South Africa, between 2006 and 2016, 2020) indicate that primary healthcare (PHC) PSA testing increased substantially in Gauteng between 2014 and 2016. It is reasonable to assume that improved access to screening in Gauteng, specifically at PHC facilities, may have resulted in the increased PCa diagnosis. Screening and education both play important roles in identifying men at risk of developing PCa. ${ }^{[8-10,31]}$

It is also possible that $\mathrm{PCa}$ awareness is a cause for the increased ASIR noted.

Table 3. APC and AAPC output from the Joinpoint model for age-standardised prostate cancer incidence rates between 2006 and 2016

\begin{tabular}{llllllll}
\hline & Segment & Lower endpoint & Upper endpoint & APC & Lower CI & Upper CI & $p$-value \\
\hline APC & 1 & 2006 & 2016 & 2.7 & -1.2 & 6.7 & $<0.0001$ \\
AAPC & 1 & 2006 & 2016 & 2.7 & -1.2 & 6.7 & $<0.0001$
\end{tabular}

$\mathrm{APC}=$ annual percentage change; $\mathrm{AAPC}=$ average annual percentage change; $\mathrm{CI}=$ confidence interval 


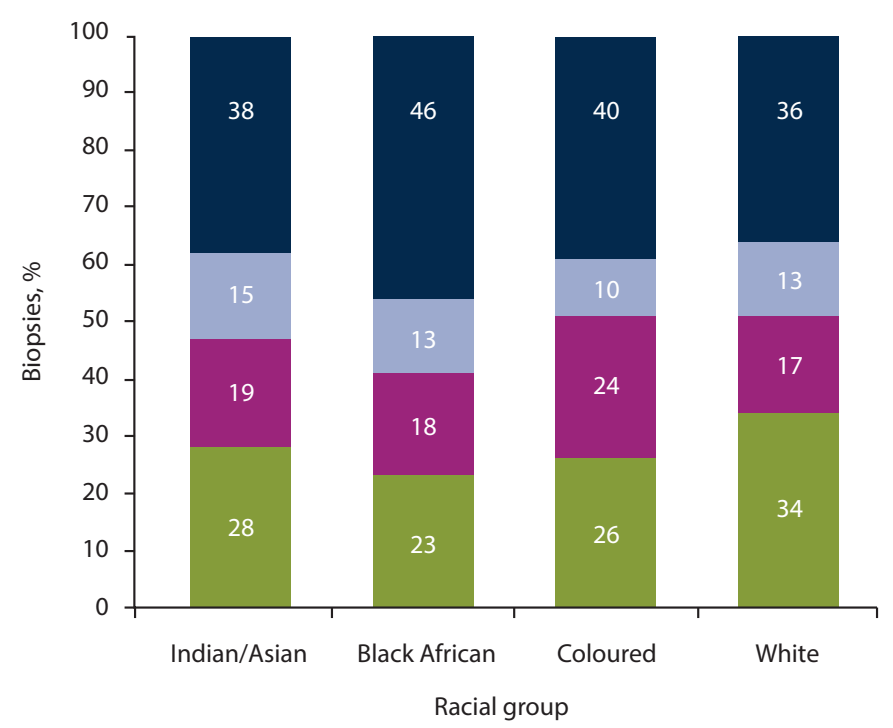

$\mathrm{GS} \leq 6(\mathrm{GG} 1)$
$\mathrm{GS} \geq 8$ (GG4 and GG5)

Fig. 3. Percentage of prostate biopsies with a low- $(G S \leq 6)$, intermediate- $(G S 7: 3+4=7$ and $4+3=$ $7)$ and high- $(G S \geq 8)$ risk GS by racial group. The GG is indicated in brackets, with 4 and 5 combined to report a $G S \geq 8$. (GS = Gleason Score; $G G=$ grade group.)

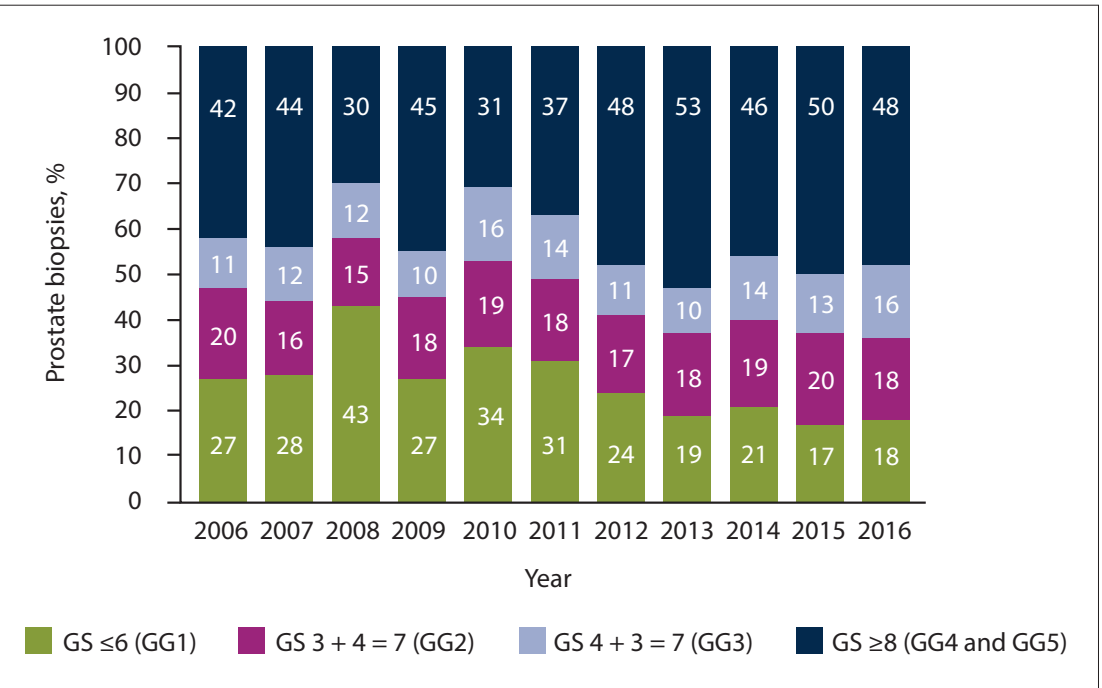

Fig. 4. Percentage of prostate biopsies with a low- $(G S \leq 6)$, intermediate- $(G S 7: 3+4=7$ and $4+3=7)$ and high- $(G S \geq 8)$ risk GS by calendar year. The GG is indicated in brackets, with 4 and 5 combined to report a $G S \geq 8$. (GS = Gleason Score; $G G=$ grade group.)

Public awareness of female cancers started to improve in 2009, promoting early detection of breast cancer with mobile mammography units. $^{[32]}$ In contrast, local awareness campaigns for PCa started much later. with initiatives such as 'Movember,', 'Daredevil Run' and 'Suit Up September' beginning in 2015 and operating predominantly in metropolitan areas. ${ }^{[33-35]}$

PCa has the highest reported heritability of any major cancer, and several genetic susceptibility loci have been identified in European and Asian men. ${ }^{[5,36]}$ Another reason for the increased ASIR may be a combination of genetic and environmental risk factors that are not known at present.

We noted an increase in the percentage of biopsies with a high-risk GS between 2006 and 2016. From 2012, a high-risk GS was reported for $\sim 50 \%$ of biopsies, highlighting late presentation in Gauteng. A system review by Ilic et al. ${ }^{[37]}$ reported that PSA testing increased the detection of early-stage disease and slightly decreased the detection of advanced PCa. Similarly, other studies have shown that access to
PSA testing has increased PCa detection rates, combined with a migration to lowerstage disease, throughout the USA, Canada and Europe. ${ }^{[38]}$ Unpublished data from our province (Chen et al.) showed that PHC PSA testing increased dramatically in 2013. We would therefore have expected to see an increase in early-stage disease (GS $\leq 6$ ). It is possible that it is too early to realise the benefit of increased PSA testing.

We noted that black African men were statistically significantly more likely than whites and coloureds to present with a GG $\geq 4$. While the reasons for this finding are uncertain, racial differences in healthcareseeking behaviour have been reported, with white and coloured respondents more likely to seek care than black Africans. ${ }^{[39]}$ In SA, black males have been shown to be less likely to access healthcare than females, and sought care first from traditional healers. ${ }^{[39]}$ Male underutilisation of healthcare services has been identified in multiple countries. ${ }^{[40]}$ One of the challenges men face is the working hours of health services. ${ }^{[40]}$ Although increasing numbers of men presented for diagnosis in our study, it may be that lack of knowledge is one of multiple factors for late presentation. ${ }^{[33-35]}$

Our finding that older age was a risk factor for high-grade $\mathrm{PCa}$ is in keeping with some studies from Canada and the USA, ${ }^{[11,42]}$ while local studies have produced conflicting results..$^{[7,12,13]}$ Although local guidelines recommend PCa screening from 40 years, we found that most patients were diagnosed in their mid-60s. ${ }^{[9,10]}$ This is similar to other local findings and published data from Ghana and Nigeria. . $^{[7,12,13,43,44]}$ Our local unpublished data (Cassim et al.) indicate that the mean age for PSA testing at PHC facilities was 55.8 years, highlighting possible delayed diagnosis. ${ }^{[30]}$ Maphayi et al. ${ }^{[45]}$ showed that only $7 \%$ of black African men who had a PSA $\geq 4 \mu \mathrm{g} / \mathrm{L}$ had a followup biopsy.

Our study builds on previous work providing evidence that laboratory data are an invaluable repository of health data. Specifically, this work has extended PCa reporting using laboratory data. Applying similar methods of reporting, such as the use of SNOMED CT lookup tables, could provide national and provincial PCa statistics across SA to complement cancer registry reporting. These approaches can be complemented and supplemented with the application of text mining and predictive analytics that have demonstrated the reliable extraction of prognostic GS data from narrative biopsy reports (Cassim N, Mapundu M, Olago V, 


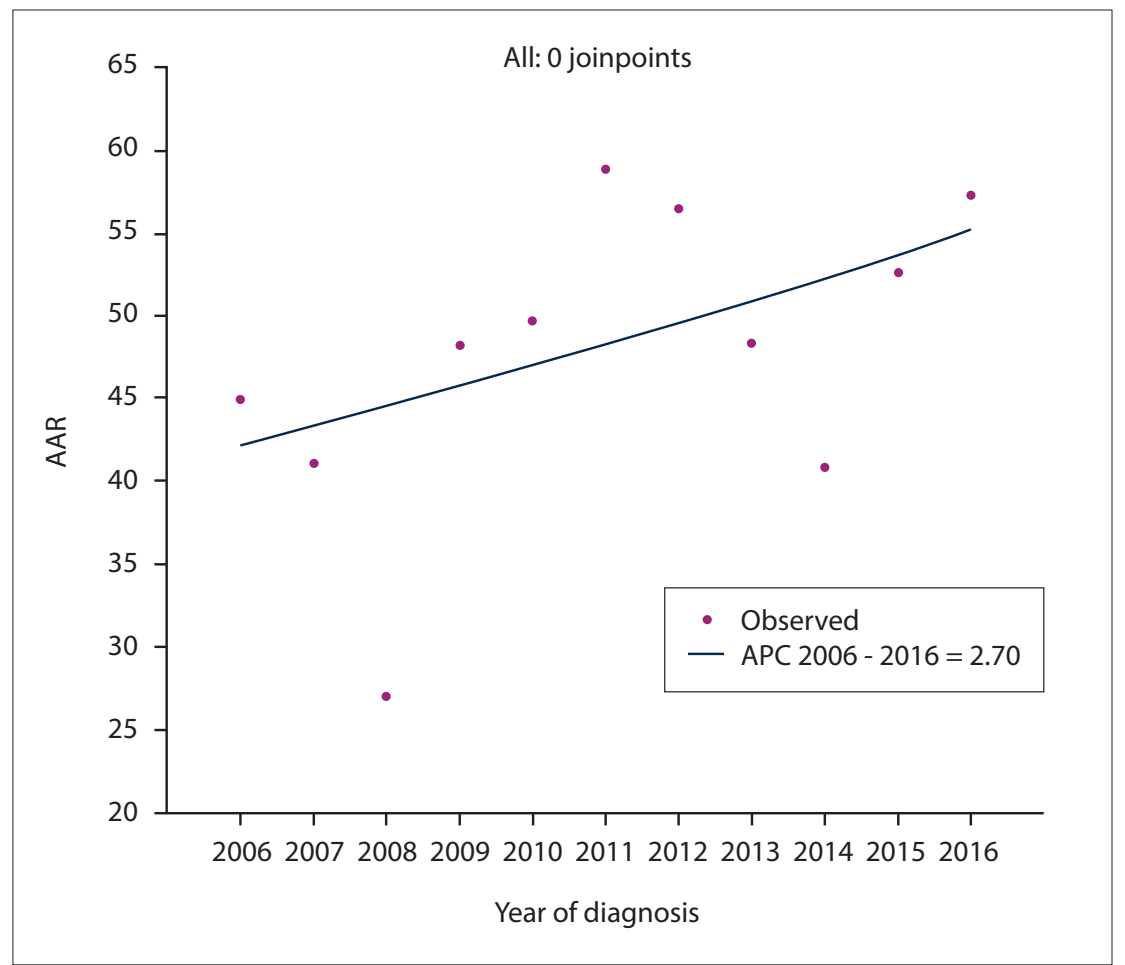

Fig. 5. Age-standardised prostate cancer incidence rates between 2006 and 2016 reported as mauve dots, with the AAPC reported as a blue line. $(A A P C=$ average annual percentage change; $A P C=$ annual percentage change; $A A R=$ age-adjusted rate. $)$

Ahmad A, George JA, Glencross DK, 'Using big data techniques to extract Gleason scores to improve prostate cancer reporting in the Gauteng province, South Africa', 2019, unpublished). These tools have the potential to generate automated PCa reporting, leading to the development of PCa epidemiological reporting using laboratory data.

\section{Study limitations}

This study did not investigate underlying contributing factors in under-performing districts as this was beyond the scope of the study. The incidence data reported for our study were reliant on the probabilistic matching algorithm for the de-duplication of patient records in the absence of a national unique patient identifier.

A limitation of our study is the absence of private sector laboratory data, affecting generalisability. There are planned discussions to receive private sector PCa data routinely. However, a local study reported that withheld private sector cancer data only accounted for $4 \%$ of cancer reporting. ${ }^{[46]}$

The accuracy of the hot-deck race imputation was also a limitation. In their unpublished study, Chen et al. used a holdout test to compare imputed versus cancer registry patient-reported racial group for 406642 unique surname-race group pairings. The historically well-populated cancer registry data were split into patient- reported racial group values and imputed values. Chen et al. compared the imputed and original values. For the imputed data set, a sensitivity of $\geq 90 \%$ was reported for black Africans, whites and Indians/Asians. However, a lower sensitivity of $63.79 \%$ was reported for coloureds. This finding indicates that the race imputation may be less accurate for coloureds, which may result in some misclassification. A challenge in SA is that common surnames are used interchangeably by coloureds and whites. ${ }^{[47]}$

It was not possible to control for confounders such as socioeconomic status and environmental exposure, as these data were not available.

\section{Conclusions}

There is a need to improve our understanding of the contribution of genetic, environmental and lifestyle factors to PCa in black African men. There is also a need to improve our understanding of late presentation in black African men. Late presentation is associated with limited treatment options and an increased mortality rate. Broader public health initiatives are required to identify men earlier by improving awareness and healthseeking behaviour as well as providing better access to urology services that meet their needs. Here, the provision of tailor-made male-only healthcare services could play an important role in reducing PCa incidence.
Declaration. The research for this study was done in partial fulfilment of the requirements for NC's PhD degree, done in the School of Pathology at the University of the Witwatersrand.

Acknowledgements. The authors thank Prof. Martin Hale, Prof. Mohammed Haffejee, Dr Innocent Maposa, Dr Elvira Singh, Dr Mazvita Sengayi and Ms Patricia Kellett for their assistance.

Author contributions. NC: executed the research, data analysis, prepared the first draft and final draft submitted. AA and RW: data analysis assistance. TRR: data analysis and editorial assistance. DKG and JAG: codeveloped and executed research, editorial input and project leader/budget owner.

Funding. This work was supported in part by grants from the US National Cancer Institute to TRR (U01-CA184374) and from the NHLS Research Trust to NC (development grant).

Conflicts of interest. None.

1. Cooperberg MR, Chan JM. Epidemiology of prostate cancer World J Urol 2017;35(6):849. https://doi.org/10.1007/s00345017-2038-0

2. Neupane S, Bray F, Auvinen A. National economic and development indicators and international variation in prostate cancer incidence and mortality: An ecological analysis. World J Urol 2017;35(6):851-858. https://doi.org/10.1007/s00345-0161953-9

3. Ferlay J, Soerjomataram I, Ervik M, et al. Cancer incidence and mortality worldwide: Sources, methods and major patterns in GLOBOCAN 2012. Int I Cancer 2015;136(5):E359-E386. https://doi.org/10.1002/ijc.29210

4. Bray F, Ferlay J, Soerjomataram I, Siegel RL, Torre LA, Jemal Bray F, Ferlay J, Soerjomataram I, Siegel RL, Torre LA, Jemal A. Global cancer statistics 2018: GLOBOCAN estimates of incidence and mortality worldwide for 36 cancers in 185 countries. CA Cancer J Clin 2018;68(6):394-424. https://doi. org/10.3322/caac. 21492

5. Rebbeck TR, Devesa SS, Chang BL, et al. Global patterns of prostate cancer incidence, aggressiveness, and mortality in men of African descent. Prostate Cancer 2013;2013:560857. https:// doi.org/10.1155/2013/560857

6. Center MM, Jemal A, Lortet-Tieulent J, et al. International variation in prostate cancer incidence and mortality rates. Eur Urol 2012;61(6):1079-1092. https://doi.org/10.1016/j. eururo.2012.02.054

7. Tindall EA, Monare LR, Petersen DC, et al. Clinical presentation of prostate cancer in black South Africans. Prostate 2014;74(8):880-891. https://doi.org/10.1002/pros.22806

8. European Association of Urology. Guidelines on Prostate European Association of Urology. Guidelines on Prostate
Cancer. Aarnheim, Netherlands: EAU, 2016 https://uroweb.org/ Cancer. Aarnheim, Netherlands: EAU, 2016 https://uroweb.org/
wp-content/uploads/EAU-Guidelines-Prostate-Cancer-2016. wp-content/uploads/EAU-Guic
pdf (accessed 19 February 2018)

pdf (accessed 19 February 2018).
9. Anderson D, Barnes R, Bida M, et al. South African Prostate Cancer Guidelines - draft version 2017. South African Urological Association, South African Society of Medical Oncology, South African Society for Clinical and Radiation Oncologists, South African Society of Nuclear Medicine, South African Oncology Consortium and Prostate Cancer Foundation of South Africa, 2017. https://prostate-ca.co.za/wp-content/uplo ads/2017ProstateGuidelinesDraftVersion2016.pdf (accessed 12 March 2017).

10. Segone AM, Haffejee M, Wentzel S, et al. Prostate Cancer Diagnostic and Treatment Guidelines: The Prostate Cancer Diagnostic and Treatment Guidelines: The Prostate Cancer
Foundation of South Africa. June 2013. http://prostate.acitravel. Foundation of South Africa. June 2013. http://prostate.acitravel.
co.za/cake/app/webroot/uploads/files/Prostate_Cancer_ co.za/cake/app/webroot/uploads/files/Prost

11. Epstein JI, Egevad L, Amin MB, et al. The 2014 International Society of Urological Pathology (ISUP) Consensus Conference on Gleason Grading of Prostatic Carcinoma: Definition of grading patterns and proposal for a new grading system. Am J Surg Pathol 2016;40(2):244-252. https://doi.org/10.1097/ PAS.0000000000000530

12. Heyns CF, Fisher M, Lecuona A, van der Merwe A. Prostate cancer among different racial groups in the Western Cape: Presenting features and management. S Afr Med J 2011;101(9):267-270

13. Le Roux HA, Urry RJ, Sartorius B, Aldous C. Prostate cancer at a regional hospital in South Africa: We are only seeing the tip of the iceberg. S Afr J Surg 2015;53(3 and 4):57-62. 
14. National Cancer Registry. Summary statistics of cancer diagnosed histologically in South Africa in 2014. Johannesburg: National Institute for Occupational Health, 2014. https://www.nicd.ac.za/wp2014. Johannesburg: National Institute for Occupational Health, 2014.
content/uploads/2019/12/2014-NCR-tables.pdf (accessed 12 May 2018).

15. National Cancer Institute at the National Institutes of Health. Understanding prostate changes: A National Cancer Institute at the National Institutes of Health. Understanding prostate changes: A
health guide for men. 2018. https://www.cancer.gov/types/prostate/understanding-prostate-changes health guide for men. 2018.
(accessed 19 February 2018)

16. National Health Laboratory Service. 2017/18 Annual Report. 2018. http://www.nhls.ac.za/assets/files/ an_report/NHLS_AR_2018.pdf (accessed 18 July 2019).

17. US National Library of Medicine. Overview of SNOMED CT. 2016. https://www.nlm.nih.gov/healthit/ snomedct/snomed_overview.html (accessed 20 February 2018)

18. Statistics South Africa. Mid-year population estimates, 2016. Statistical release P0302. Pretoria: Stats SA, 2016. https://www.statssa.gov.za/publications/P0302/P03022016.pdf (accessed 17 June 2018).

19. Statistics South Africa. Mid-year population estimates, 2020. Statistical release P0302. Pretoria: Stats SA, 2020. http://www.statssa.gov.za/publications/P0302/P03022020.pdf (accessed 23 July 2018).

20. Oosthuizen M, Naidoo P. Internal migration to the Gauteng Province. Development Policy Research Unit, University of Cape Town, 1 December 2004. https://www.africaportal.org/publications/internalmigration-to-the-gauteng-province/ (accessed 7 February 2019).

21. Ngobeni T. Corporate DataWarehouse patient linking technical document. Johannesburg: National Health Laboratory Service, 2017.

22. International Agency for Research on Cancer, World Health Organisation. International Classification of Diseases for Oncology. 3rd ed. Geneva: WHO, 2013. https://apps.who.int/iris/bitstream/ handle/10665/96612/9789241548496 eng.pdf (accessed 25 April 2018).

23. Singh E, Greyling M. A review of hot deck imputation for survey non responses: The example of population group in the national cancer registry database. Johannesburg: National Institute for population group in the nat

24. Statistics South Africa. Census 2011: Key results. 2011. http://www.statssa.gov.za/census/census_2011/ census_products/Census_2011_Key_results.pdf (accessed 23 January 2019).

25. FamilyTreeDNA. South African Cape Coloured. https://www.familytreedna.com/groups/capecoloured-dna-project/surnames (accessed 31 July 2018).

26. Gauteng Department of Health. Annual Report: 2013/14. Johannesburg: GDOH, 2017. https:// cmbinary.gauteng.gov.za/Media?path=cphealth/Documents/Annual\%20Reports/Department $\% 20$ of\%20Health\%20Annual\%20Report\%202014-2015.pdf\&Item=331\&Type=Documents\&Location=/ cphealth (accessed 11 March 2019).

27. Babb C, Urban M, Kielkowski D, Kellett P. Prostate cancer in South Africa: Pathology based national cancer registry data (1986 - 2006) and mortality rates (1997 - 2009). Prostate Cancer 2014;2014:419801. https://doi.org/10.1155/2014/419801

28. National Cancer Registry. Summary Statistics of Cancer Diagnosed Histologically in South Africa in 2007. Johannesburg: National Institute for Occupational Health, 2007. https://www.nicd.ac.za/wpcontent/uploads/2019/12/2007-NCR-2007-results.pdf (accessed 12 May 2018).

29. National Cancer Registry. Summary statistics of cancer diagnosed histologically in South Africa in 2013. Johannesburg: National Institute for Occupational Health, 2013. https://www.nicd.ac.za/wpcontent/uploads/2019/12/2013-NCR.pdf (accessed 12 May 2018).

30. National Cancer Registry. Summary statistics of cancer diagnosed histologically in South Africa in 2012. Johannesburg: National Institute for Occupational Health, 2012. https://www.nicd.ac.za/wp2012. Johannesburg: National Institute for Occupational Health, 2012. https://Ww
content/uploads/2019/12/2012-NCR-2012-results.pdf (accessed 26 February 2018).
31. Mndzebele SL, Mogale JW. Prostate cancer awareness and screening behaviour among men in Gauteng Province, South Africa: Utilization of primary health care services. Afr J Phys Health Educ Recreation Dance 2015;21(Suppl 2):296-305

32. Pink Drive. Pink Drive: TLC Ladies MTB and Trail Running Weekend. 2016. Available https:// www.pinkdrive.co.za/event/tlc-ladies-mtb-and-trail-running-weekend/http://www.pinkdrive.co.za/ (accessed 30 August 2016).

33. The Men's Foundation. Movember. 2018. https://za.movember.com/story/11810/highlights-of movember-2018 (accessed 28 August 2018)

34. Hollard. Hollard Daredevil Run. 2018. https://www.daredevilrun.com/ (accessed 28 August 2018).

35. Prostate Cancer Foundation. Suit Up September - Prostate Cancer Awareness Campaign. 2017. http:/ www.prostate-ca.co.za/cake/index.php/article/76 (accessed 28 August 2018).

36. Rebbeck TR. Prostate cancer genetics: Variation by race, ethnicity, and geography. Semin Radiat Oncol 2017;27(1):3-10. https://doi.org/10.1016/.j.semradonc.2016.08.002

37. Ilic D, Djulbegovic M, Jung JH, et al. Prostate cancer screening with prostate-specific antigen (PSA) test: A systematic review and meta-analysis. BMJ 2018;362:k3519. https://doi.org/10.1136/bmj.k3519

38. Thompson IM, Ankerst DP. Prostate-specific antigen in the early detection of prostate cancer. Can Med Assoc J 2007;176(13):1853-1858. https://doi.org/10.1503/cmaj.060955

39. Abera Abaerei A, Ncayiyana J, Levin J. Health-care utilization and associated factors in Gauten province, South Africa. Glob Health Action 2017;10(1):1305765. https://doi.org/10.1080/16549716.2 017.1305765

40. Baker P, Shand T. Men's health: Time for a new approach to policy and practice? J Glob Health 2017;7(1). https://doi.org/10.7189/jogh.07.010306

41. Bechis SK, Carroll PR, Cooperberg MR. Impact of age at diagnosis on prostate cancer treatment and survival. J Clin Oncol 2011;29(2):235-241. https://doi.org/10.1200/Jco.2010.30.2075

42. Alibhai SMH, Krahn MD, Fleshner NE, Cohen MM, Tomlinson GA, Naglie G. The association between patient age and prostate cancer stage and grade at diagnosis. BJU Int 2004;94(3):303-306. https://doi.org/10.1111/j.1464-410X.2004.04883.x

43. Muhammad A, Abdulwahab-Ahmed A, Agwu N, Yunusa G, Abdullahi K, Mungadi I. Use of transrectal ultrasound in the management of urologic diseases in a tertiary hospital in north-western Nigeria. 2018;25(3):172-176. https://doi.org/10.4103/npmj.npmj_131_18

Yeboah ED, Hsing AW, Mante S, et al. Management of prostate cancer in Accra, Ghana. J West Afr Coll Surg 2016;6(4):31-65.

45. Maphayi M, Cassim N, Bello B, George JA. Mining laboratory data to describe prostate specific antigen testing and prostate cancer in Johannesburg, South Africa. Pan Afr Med J 2020;35:61. https://doi. org/10.11604/pamj. 2020.35.61.21331

46. Singh E, Underwood JM, Nattey C, Babb C, Sengayi M, Kellett P. South African National Cancer Registry: Effect of withheld data from private health systems on cancer incidence estimates. S Afr Med J 2015;105(2):107-109. https://doi.org/10.7196/SAMJ.8858

47. Snell R. Cape slave naming patterns. South African History Online, 2 August 2016. https://www sahistory.org.za/archive/cape-slave-naming-patterns-robert-shell (accessed 12 March 2018).

Accepted 19 June 2020. 